\title{
Tricuspid Valve
}

National Cancer Institute

\section{Source}

National Cancer Institute. Tricuspid Valve. NCI Thesaurus. Code C12805.

A three-cusp valve of the heart that regulates the flow of blood between the right atrium and the right ventricle of the heart. 